\title{
ON RINGS OF BOUNDED CONTINUOUS FUNCTIONS WITH VALUES IN A DIVISION RING
}

\author{
ELLEN CORREL AND MELVIN HENRIKSEN ${ }^{1}$
}

Introduction. Let $C^{*}(X, A)$ denote the ring of bounded continuous functions on a (Hausdorff) topological space $^{2} X$ with values in a topological division ring $A$. If, for every maximal (two-sided) ideal $M$ of $C^{*}(X, A)$, we have $C^{*}(X, A) / M$ is isomorphic with $A$, we say that Stone's theorem holds for $C^{*}(X, A)$. It is well known $[9 ; 6]$ that Stone's theorem holds for $C^{*}(X, A)$ if $A$ is locally compact and connected, or a finite field. In giving a partial answer to a question of Kaplansky [7], Goldhaber and Wolk showed in [5] that, with restriction on $X$, and if $A$ is of type $V$ and satisfies the first axiom of countability, then a necessary condition that Stone's theorem holds for $C^{*}(X, A)$ is that $A$ be locally compact. They ask if this condition is also sufficient.

Below, we answer this question in the affirmative.

In the course of their proof of the necessity of this condition, they construct a class of maximal ideals of $C^{*}(X, A)$. (See $\S 3$ ). They also ask if every maximal ideal of $C^{*}(X, A)$ is in this class.

We show that even if both $X$ and $A$ are the reals, the answer to this question is no.

1. Background material. Let $A$ denote a topological division ring (with continuous inversion). If $B, C$ are subsets of $A$, let $B C=\{b c: b \in B, c \in C\}$, and if $0 \in B$, let $B^{-1}=\left\{b^{-1}: b \in B\right\}$.

A subset $P$ of $A$ is said to be bounded if for every neighborhood $U$ of 0 , there is a neighborhood $V$ of 0 such that $P V$ and $V P$ are contained in $U$. It is easily seen that every compact ${ }^{2}$ subset of $A$ is bounded [7].

If $A$ is locally compact, then bounded closed subsets are compact. If $A$ is of type $V$, the converse is also true [5].

In fact, the structure of locally compact (nondiscrete) division rings is well known (see, for example, [8]). The only connected locally

Presented to the Society, November 27, 1954; received by the editors May 21, 1955.

1 This author was supported (in part) by the National Science Foundation, Contract No. NSF-G1129.

2 All topological spaces considered are assumed to be Hausdorff. $X$ is called compact if every open covering has a finite subcovering; countably compact if every countable open covering has a finite subcovering. Goldhaber and Wolk [5] use "sequentially compact" for this latter term. 
compact division rings are the real field $R$, the complex field $K$, and the division algebra $Q$ of real quaternions. In the totally disconnected (nondiscrete) case, they consist of the $p$-adic fields, the fields of formal power series over a finite field, or division algebras of finite order over one of these fields. We shall not, however, use this explicit characterization in the totally disconnected case.

Let $C(X, A)$ denote the ring of all continuous $A$-valued functions on a topological space $X$. An element $f$ of $C(X, A)$ is said to be bounded if its range $r(f)$ is a bounded subset of $A$. It is easily seen [7] that the set of all bounded functions of $C(X, A)$ forms a subring $C^{*}(X, A)$ of $C(X, A)$.

We say that $X$ is completely regular with respect to $A$ if for every $x \in X$ and every neighborhood $U$ of $x$, there is an $f \in C^{*}(X, A)$ such that $f(x)=0, f(X-U)=a \neq 0$. (Compare with [5]). If $A=R$ (the real line), this coincides with the usual notion of complete regularity. It is easy to show that $X$ is completely regular with respect to a totally disconnected division ring if and only if $X$ has a basis of open and closed sets (i.e., $X$ is 0 -dimensional).

It is easily seen $[1$, p. 32, Ex. $15 ;$ p. 56, Ex. 6] that the set of functions in $C(X, A)$, the closure of whose range is compact, forms a subring of $C^{*}(X, A)$, which we denote by $C p(X, A)$.

Every completely regular space $X$ can be imbedded in an essentially unique compact space $\beta X$, called the Stone-Cech compactification of $X$, such that (a) $X$ is dense in $\beta X$ and (b) every $f \in C^{*}(X, R)$ has an (unique) extension over $\beta X[9 ; 2]$. Thus, $C^{*}(X, R)$ and $C(\beta X, R)=C^{*}(\beta X, R)$ are isomorphic.

A completely regular space $X$ is called pseudo-compact [6] if $C(X, R)=C^{*}(X, R)$. Every normal pseudo-compact space is countably compact, ${ }^{2}$ but there are non-normal pseudo-compact spaces that are not countably compact (see Example 1).

Stone's theorem is said to hold for a subring $C^{\prime}$ of $C(X, A)$ if for every maximal (two-sided) ideal $M$ of $C^{\prime}$, we have $C^{\prime} / M$ is isomorphic with $A$. It is well known $[9 ; 6]$ that Stone's theorem holds for $C^{*}(X, A)$ when $A$ is $R, K$, or $Q$ (i.e., when $A$ is locally compact and connected), or when $A$ is a finite field.

2. Stone's theorem for $C p(X, A)$. We give now the main theorem of this paper.

Theorem 1. If $X$ is completely regular, and $A$ is a totally disconnected division ring, then Stone's theorem holds for $C_{p}(X, A)$.

Proof. Every $f \in C p(X, A)$ is a continuous mapping of $X$ into the closure $\bar{r}(f)$ of the range of $f$. By hypothesis $\bar{r}(f)$ is compact, so, by a 
theorem of Stone [9, Theorem 88], $f$ has a continuous extension over $\beta X$ into $\bar{r}(f)$. Since a continuous image of a compact space is compact, it follows that $C p(X, A)$ and $C(\beta X, A)$ are isomorphic.

Let $(\beta X)^{*}$ denote the space of components of $\beta X$ in the quotient space topology. By $[2$, p. 165$],(\beta X)^{*}$ is a compact totally disconnected space. Since $A$ is totally disconnected, every $f \in C(\beta X, A)$ is constant on each component of $\beta X$. Hence, $C(\beta X, A)$ and $C\left((\beta X)^{*}, A\right)$ are isomorphic.

By a theorem of Kaplansky [7, Theorem 24], for each maximal ideal $M$ of $C\left((\beta X)^{*}, A\right)$, there is a $p \in(\beta X)^{*}$ such that $M=\left\{f \in C\left((\beta X)^{*}, A\right): f(p)=0\right\}$. Clearly the correspondence $f \rightarrow f(p)$ is a homomorphism of $C\left((\beta X)^{*}, A\right)$ upon $A$ whose kernel is $M$.

Corollary. If (a) $X$ is completely regular and $A$ is nondiscrete, locally compact, and totally disconnected, or if (b) $X$ is pseudo-compact, and $A$ is metric, and totally disconnected, then Stone's theorem holds for $C^{*}(X, A)$.

Proof. In either case, $C^{*}(X, A)=C p(X, A)$ : in (a), since bounded closed sets are compact (see $\$ 1)$; in (b), since a continuous image of a pseudo-compact space is pseudo-compact, and since a pseudo-compact subspace of a metric space is compact [7].

In view of this corollary and the known facts about connected locally compact division rings given in $\$ 1$, we have an affirmative answer to the first question of Goldhaber and Wolk (see the Introduction and $\S 3$ ).

THEOREM 2. If $X$ is completely regular and $A$ is a nondiscrete locally compact division ring, then Stone's theorem holds for $C^{*}(X, A)$.

3. The Goldhaber-Wolk theorem. If $X$ is not countably compact, ${ }^{2}$ let $S$ be a family of closed, countably infinite, discrete subsets of $X$, maximal with respect to being closed under finite intersection, and let $M(S)=\left\{f \in C^{*}(X, A): f \rightarrow 0\right.$ on some element of $\left.S\right\}$. Goldhaber and Wolk [5] state that if (i) $X$ is completely regular with respect to $A$, and (ii) $A$ is of type $\mathrm{V}$ and satisfies the first axiom of countability, then $M(S)$ is a maximal ideal of $C^{*}(X, A)$, and moreover,

$$
C^{*}(X, A) / M(S)
$$

is isomorphic with $A$ if and only if $A$ is locally compact.

Their proof relies on a lemma [5, Lemma 2], which states that if (i) holds, then every bounded $A$-valued function on a closed, countable, discrete subset of $X$ has a bounded continuous extension over $X$. 
Actually (i) is not sufficiently strong to insure this conclusion. ${ }^{8}$ In fact, unless (i) is strengthened, the Goldhaber-Wolk theorem is not correct. If, however, we assume in addition that $X$ is normal, then both this lemma and the Goldhaber-Wolk theorem become valid. ${ }^{4}$

We give below two examples of non-normal spaces to justify these assertions.

For any ordinal $\alpha$, let $W(\alpha)$ denote the space of ordinals $\leq \alpha$ with the interval topology. As usual, $\omega$ denotes the first infinite ordinal and $\omega_{1}$ denotes the first uncountable ordinal.

ExAmple 1. Let $T=W(\omega+1) \times W\left(\omega_{1}+1\right)-\left\{\left(\omega, \omega_{1}\right)\right\}$. It is well known that $T$ is a non-normal pseudo-compact space that is not countably compact [6]. Moreover, if $A$ is the rational field, any $f \in C^{*}(X, A)$ must be constant on all but a finite subset of the closed, countable discrete subspace $N=\left\{\left(n, \omega_{1}\right): n<\omega\right\}$ of $T$, or $f(N)$ has a unique limit point. Moreover, by the corollary to Theorem 1, Stone's theorem holds for $C^{*}(T, A)$.

EXAMPLE 2. Let $W^{\prime}\left(\omega_{1}+1\right)$ be the space obtained by modifying the topology of $W\left(\omega_{1}+1\right)$ as follows: each $\alpha<\omega_{1}$ is isolated, while neighborhoods of $\omega_{1}$ are as before.

Let $T^{\prime}=W(\omega+1) \times W^{\prime}\left(\omega_{1}+1\right)-\left\{\left(\omega, \omega_{1}\right)\right\}$. It is easily seen that $T^{\prime}$ is completely regular, non-normal, and not pseudo-compact (whence not countably compact). Again, any $f \in C^{*}(X, A)$, for $A$ the rationals, is constant for all but a finite subset of the closed, countable, discrete subset $N$ of $T^{\prime}$, or $f(N)$ has a unique limit point.

Let $S$ be a family of infinite subsets of $N$ maximal with respect to being closed under finite intersection. Let $S^{\prime}$ be the family of all closed, countable, discrete subsets of $T^{\prime}$ that contain an element of $S$. It is easily seen that $S^{\prime}$ is maximal with respect to being closed under finite intersection, and that $M\left(S^{\prime}\right)$ is a maximal ideal of $C^{*}\left(T^{\prime}, A\right)$. Moreover, it is easy to verify that $C^{*}\left(T^{\prime}, A\right) / M\left(S^{\prime}\right)$ and $A$ are isomorphic. However, Stone's theorem does not hold for $C^{*}\left(T^{\prime}, A\right)$.

Goldhaber and Wolk also ask if every maximal ideal of $C^{*}(X, A)$ necessarily takes the form $M(S)$. The answer is no, even if $X=A=R$, as is shown by the following modification of an example in [4].

EXAmple 3. Let $\mu$ denote Lebesgue measure on the real line and, for any $f \in C^{*}(R, R)$ let $Z(f)=\{x \in R: f(x)=0\}$. Let $I=\left\{f \in C^{*}(R, R)\right.$ :

3 We are indebted to L. Gillman for pointing this out to us.

- Let $X$ be such that if $\left\{x_{n}\right\}_{n=1}^{\infty}$ is a closed, countable discrete subspace of $X$, there is an $f \in C(X, R)$ such that $f\left(x_{n}\right)=n$. (This will certainly hold if $X$ is normal). Let $U_{n}=\{x \in X: n-1 / 2<f(x)<n+1 / 2\}$. Then $U_{n}$ is a neighborhood of $X_{n}$, the $U_{n}$ 's are pairwise disjoint, and the union of any subcollection of their closures is closed. With this addition, the remainder of the Goldhaber-Wolk argument is valid. 
$\mu(R-Z(f))<\infty\}$. Clearly $I$ is a (proper) ideal of $C^{*}(R, R)$.

If $M$ is any maximal ideal of $C^{*}(R, R)$ containing $I$, and if $D$ is any closed, countable, discrete subset of $R$, we shall show that there is a $g \in I \subset M$ such that $g$ does not approach 0 on $D$. Let $V$ be any open set of finite measure containing $D$. There is a $g \in C^{*}(R, R)$ such that $g(D)=1$, and $g(R-V)=0$. Since $R-Z(g) \subset V$, and $\mu(V)<\infty$, we have $g \in I \subset M$. Thus, $M$ cannot be of the form $M(S)$.

Added October 24, 1955. Recently, B. Banaschewski (Math. Nachr. vol. 13 (1955) pp. 129-140) has shown that if a 0-dimensional space $X$ is dense in a compact 0 -dimensional space $B$, there is a continuous map of $(\beta X)^{*}$ (see the proof of Theorem 1 above) onto $B$ keeping $X$ pointwise fixed. Since for a totally disconnected division ring $A$, every $f \in C p(X, A)$ has an extension over $(\beta X)^{*}$, this space is the analogue for $A$-valued functions of the Stone-Cech compactification $\beta X$.

He also asks if there are 0 -dimensional spaces for which $(\beta X)^{*} \neq \beta X$. Two such examples of normal spaces are given by $C$. $H$. Dowker (Quart. J. Math. vol. 6 (1955) pp. 101-120, Examples M and P), and another (not necessarily normal) by J. R. Isbell (Tôhoku Math. J. vol. 7 (1955) pp. 1-8.)

Added in proof. Since the preparation of this article, the GoldhaberWolk problems have been solved independently by H. J. Kowalsky (Mathematische Nachrichten vol. 14 (1955) pp. 57-64).

\section{REFERENCES}

1. N. Bourbaki, Groupes topologiques, Actualités Scientifiques et Industrielles, no. 1143, Paris, 1951.

2. - Structures uniformes, Actualités Scientifiques et Industrielles, no. 1142, Paris, 1951.

3. E. Cech, On bicompact spaces, Ann. of Math. vol. 38 (1937) pp. 823-844.

4. P. Erdös, L. Gillman, and M. Henriksen, $A n$ isomorphism theorem for real-closed fields, Ann. of Math. vol. 61 (1955) pp. 542-554.

5. J. K. Goldhaber and E. S. Wolk, Maximal ideals in rings of bounded continuous functions, Duke Math. J. vol. 21 (1954) pp. 565-569.

6. E. Hewitt, Rings of real-valued continuous functions. I, Trans. Amer. Math. Soc. vol. 64 (1948) pp. 45-99.

7. I. Kaplansky, Topological rings, Amer. J. Math. vol. 69 (1947) pp. 153-183.

8. , Topological rings, Bull. Amer. Math. Soc. vol. 54 (1948) pp. 809-826.

9. M. H. Stone, Applications of the theory of Boolean rings to general topology, Trans. Amer. Math. Soc. vol. 41 (1937) pp. 375-481.

Purdue University 